\title{
Characterization of type-2 diacylglycerol acyltransferases in Haematococcus lacustris reveals their functions and engineering potential in triacylglycerol biosynthesis
}

Hongli Cui ${ }^{\dagger}$, Chunchao Zhao ${ }^{\dagger}$, Wenxin Xu, Hongjiang Zhang, Wei Hang, Xiaoli Zhu, Chunli Ji, Jinai Xue, Chunhui Zhang and Runzhi Li*

\begin{abstract}
Background: Haematococcus lacustris is an ideal source of astaxanthin (AST), which is stored in oil bodies containing esterified AST (EAST) and triacylglycerol (TAG). Diacylglycerol acyltransferases (DGATs) catalyze the last step of acyl-CoA-dependent TAG biosynthesis and are also considered as crucial enzymes involved in EAST biosynthesis in $\mathrm{H}$. lacustris. Previous studies have identified four putative DGAT2-encoding genes in $\mathrm{H}$. lacustris, and only HpDGAT2D allowed the recovery of TAG biosynthesis, but the engineering potential of HpDGAT2s in TAG biosynthesis remains ambiguous.

Results: Five putative DGAT2 genes (HpDGAT2A, HpDGAT2B, HpDGAT2C, HpDGAT2D, and HpDGAT2E) were identified in H. lacustris. Transcription analysis showed that the expression levels of the HpDGAT2A, HpDGAT2D, and HpDGAT2E genes markedly increased under high light and nitrogen deficient conditions with distinct patterns, which led to significant TAG and EAST accumulation. Functional complementation demonstrated that HpDGAT2A, HpDGAT2B, HpDGAT2D, and HpDGAT2E had the capacity to restore TAG synthesis in a TAG-deficient yeast strain (H1246) showing a large difference in enzymatic activity. Fatty acid (FA) profile assays revealed that HpDGAT2A, HpDGAT2D, and HpDGAT2E, but not HpDGAT2B, preferred monounsaturated fatty acyl-CoAs (MUFAs) for TAG synthesis in yeast cells, and showed a preference for polyunsaturated fatty acyl-CoAs (PUFAs) based on their feeding strategy. The heterologous expression of HpDGAT2D in Arabidopsis thaliana and Chlamydomonas reinhardtii significantly increased the TAG content and obviously promoted the MUFAs and PUFAs contents.

Conclusions: Our study represents systematic work on the characterization of HpDGAT2s by integrating expression patterns, AST/TAG accumulation, functional complementation, and heterologous expression in yeast, plants, and algae. These results (1) update the gene models of HpDGAT2s, (2) prove the TAG biosynthesis capacity of HpDGAT2s, (3) show the strong preference for MUFAs and PUFAs, and (4) offer target genes to modulate TAG biosynthesis by using genetic engineering methods.
\end{abstract}

Keywords: Haematococcus lacustris, Diacylglycerol acyltransferase, Function characterization, Triacylglycerol, Genetic engineering

\footnotetext{
* Correspondence: rli2001@126.com

${ }^{\dagger}$ Hongli Cui and Chunchao Zhao contributed equally to this work. College of Agriculture, Institute of Molecular Agriculture and Bioenergy, Shanxi Agricultural University, Taigu 030801, Shanxi, China
}

(c) The Author(s). 2020 Open Access This article is licensed under a Creative Commons Attribution 4.0 International License, which permits use, sharing, adaptation, distribution and reproduction in any medium or format, as long as you give appropriate credit to the original author(s) and the source, provide a link to the Creative Commons licence, and indicate if changes were made. The images or other third party material in this article are included in the article's Creative Commons licence, unless indicated otherwise in a credit line to the material. If material is not included in the article's Creative Commons licence and your intended use is not permitted by statutory regulation or exceeds the permitted use, you will need to obtain permission directly from the copyright holder. To view a copy of this licence, visit http://creativecommons.org/licenses/by/4.0/. The Creative Commons Public Domain Dedication waiver (http://creativecommons.org/publicdomain/zero/1.0/) applies to the data made available in this article, unless otherwise stated in a credit line to the data. 


\section{Background}

Triacylglycerol (TAG) is the principal energy storage form in eukaryotic organisms and represents a promising source of biodiesel production [1]. Microalgae can efficiently absorb $\mathrm{CO}_{2}$ in the atmosphere and turn it into abundant high-value products, including polysaccharides, lipids, proteins, pigments, and biofuels [2-5]. Due to their high photosynthetic efficiency, rapid reproduction rate, and short growth cycle, microalgae have been considered as the best candidates to resolve energy crises and environmental pollution [6]. Further understanding of the pathways and regulatory mechanisms involved in TAG accumulation will facilitate the genetic engineering of microalgae [7-9].

Generally, TAG biosynthesis takes place in the endoplasmic reticulum, and TAG assembly can be divided into acyl-CoA-dependent and acyl-CoA independent pathways [10]. Diacylglycerol acyltransferases (DGATs) catalyze the final acylation of sn-1, 2-diacylglycerol (DAG) to form TAG, which is the last and limiting step in the acyl-CoA dependent TAG formation pathway [11]. These enzymes represent a bottleneck in TAG biosynthesis in some oilseed crops and algae, and thus have been regarded as key targets for manipulating TAG production [11]. In higher plants and microalgae, there are four major groups of DGATs: (1) the membrane bound form of DGAT1, (2) the membrane bound form of DGAT2 sharing low sequence similarity with DGAT1, (3) the soluble type of DGAT3, which is localized in the cytosol, and (4) the dual function of WS/DGAT, which possesses both wax ester and TAG biosynthesis activities [12-18]. DGAT1s play a critical role in TAG accumulation in many higher plants and microalgae, whereas DGAT2s appear to have an important role in the formation of TAGs containing unusual fatty acids (FAs) [14]. There is strong evidence supporting the involvement of DGAT3 and WS/DGAT in TAG biosynthesis in microalgae $[15,16]$. Usually, only one or two alleles of DGAT1s are identified in a number of microalgae, whereas multiple alleles of DGAT2s are typically present, suggesting that DGAT2s may have an important function in TAG biosynthesis [12-14, 19-27]. Recently, most of the current knowledge about algal DGATs is derived from limited algal species, including Chlamydomonas reinhardtii, Chlorella ellipsoidea, Nannochloropsis oceanica, Lobosphaera incise, Chlorella/Chromochloris zofingiensis, Myrmecia incise, and Phaeodactylum tricornutum, in which DGATs have been manipulated for molecular cloning, biochemical identification, functional characterization, and to assess their engineering potential for modulating TAG biosynthesis [19-28]. Interestingly, diverse microalgae are prominent candidates for DGATs, and the functions of distinct DGATs are unique or species-specific. Therefore, DGATs in other industrially relevant astaxanthin (AST)-producing algae, such as Haematococcus lacustris, have garnered research interest [29].
$H$. lacustris is a green microalga widely known for its ability to synthesize the highest amount of AST (4\% dry weight) under stress conditions [29, 30]. Natural AST is a red-coloured keto-carotenoid with strong antioxidant ability and important commercial value [31]. Interstingly, under environmental stress, TAG accumulation is concomitant with AST accumulation, which accumulates after biosynthesis from zeaxanthin and canthaxanthin, and is stored in oil bodies containing esterified AST (EAST) and triacylglycerol (TAG) in H. lacustris [32-35]. Moreover, previous studies have indicated that the main forms of EAST include monoester AST (M-AST, 70\%) and diester AST (D-AST, 25\%) [36-40]. Although the exact mechanisms of stress-induced TAG and AST accumulation in $H$. lacustris are not well understood, several lines of evidence have suggested that the biosynthesis of both compounds appears to be linked through the regulation of oil biosynthetic enzymes at the transcription level [40]. Indeed, the accumulation of AST appears to be dependent on the biosynthesis of FAs and accumulation of TAG [34, 41]. In addition, it has been speculated that certain DGATs are candidate enzymes catalyzing the esterification of AST in $H$. pluvialis [34]. Recently, although four putative type-2 DGATs (HpDGAT2A, $H p D G A T 2 B, H p D G A T 2 D$, and $H p D G A T 2 E$ ) were identified from $H$. pluvialis (lacustris), and only HpDGAT2D had the capability of to restore TAG biosynthesis in a TAG-deficient yeast strain [42], the engineering potential of DGAT2s in TAG biosynthesis remains ambiguous.

By employing the industrially relevant AST-producing alga $H$. lacustris, in the present study, we present systematic work on the characterization of HpDGAT2s by integrating expression patterns, AST/TAG accumulation, functional complementation, and heterologous expression in yeast, plants, and algae. Five putative $H p D G A T 2 s$ were identified in $H$. lacustris, of which, the transcription levels of $H p D G A T 2$ genes markedly increased under high light (HL) and nitrogen deficient (ND) conditions with distinct patterns, which led to significant TAG and EAST accumulation. HpDGAT2A, HpDGAT2D, and HpDGAT2E rather than HpDGAT2B had strong TAG biosynthesis activity and preferred monounsaturated fatty acyl-CoAs (MUFAs) and polyunsaturated fatty acyl-CoAs (PUFAs). Overexpression experiments indicated the engineering potential of HpDGAT2D in modulating TAG accumulation and FAs composition in algae and plants.

\section{Results}

\section{Molecular cloning and bioinformatics analysis of HpDGAT2 genes}

Based on the $H$. lacustris transcriptome database [43], five putative DGAT2 genes were predicted by the BLAST method using other DGAT2s from different algal species (Additional file 1: Table S1) as queries. The full-length 
mRNA sequences of the five genes were obtained by the rapid amplification of cDNA ends (RACEs) method, and the initiation codon, termination codon, $5^{\prime}$-untranslated region (5'-UTR), 3'-untranslated region ( $3^{\prime}$-UTR), and poly (A) characteristic tail were determined. Five putative DGAT2 genes were designed, HpDGAT2A, HpDGAT2B, HpDGAT2C, HpDGAT2D, and HpDGAT2E, by multiple sequence alignment with $C r D G A T 2 s$, four of which, HpDGAT2A, HpDGAT2B, HpDGAT2D, and HpDGAT2E, contained a full-length open reading frame (ORF), while HpDGAT2C was a partial sequence (Additional file 2: Table S2 and Additional file 3: Table S3). Then, the fulllength ORFs were cloned and sequenced by PCR with primers (Additional file 4: Table S4), which were renamed and deposited in NCBI GenBank (HpDGAT2A: MT875161; HpDGAT2B: MT875162; HpDGAT2C: MT875163; HpDGAT2D: MT875164; HpDGAT2E: MT875165). To date, this is the highest dose of DGAT2s reported in the green alga $H$. lacustris. Based on a comparison with gene models of $H p D G A T 2 s$ reported by Nguyen et al. [42], our results confirmed that there were five HpDGAT2s members in H. lacustris. Generally, only one or two alleles of DGAT1s are identified in a number of microalgae, whereas multiple alleles of DGAT2s are typically present [14].

To gain insights into the biochemical characteristics of HpDGAT2s, the molecular weight (MW), isoelectric point (pI), subcellular location, transmembrane domain (TM), signal peptide (SP), chloroplast transfer peptide (CTP), and phosphorylation site (Phos) were analyzed. No SP or CTP was present in HpDGAT2s protein sequences except for CTP in HpDGAT2C (Additional file 2: Table S2). There were two TMs in all pDGAT2s protein sequences except for three TMs in HpDGAT2B (Additional file 2: Table S2 and Additional file 5: Fig. S1), which is consistent with the membrane bound forms of DGAT1 and DGAT2 [14]. In addition, 14-30 phosphorylation sites were predicted in HpDGAT2 protein sequences (Additional file 2: Table S2 and Additional file 6: Fig. S2), indicating that phosphorylation plays important roles in DGAT2 enzyme activity because DGAT1 enzyme activity is affected by serine phosphorylation sites in mouse DGAT1 [44], TmDGAT1 [45], and BnDGAT1 [46]. It remains to be determined whether these phosphorylation sites are important for the functional regulation of HpDGAT2 in vivo.

To further analyze the conserved domains (CDs) and evolutionary relationship between HpDGAT2s and other algal DGAT2s, multiple sequence alignment and a phylogenetic tree were reconstructed. CDs analysis showed that HaeDGAT2s contained 7 CDs [26, 47, 48], including YF/YFP block (CD1), which is essential for DGAT2 activity; HPHG/EPHS block (CD2), which is proposed to partially consist of the active site; PxxR ( $\mathrm{x}=$ random amino acid) block (CD3); xGGxAE block (CD4);
$\operatorname{RxGFx}(\mathrm{K} / \mathrm{R}) \mathrm{xAxxxGxx}(\mathrm{L} / \mathrm{V})$ VPxxxFG block (CD5), which is the longest conserved sequence in plants and animals; PxxxVVGxPIxVP block (CD6); and RHK block (CD7) (Additional file 7: Fig. S3). As shown in Additional file 7: Fig. S3, there were two completely conserved amino acid residues (proline, $\mathrm{P}$ and phenylalanine, F) among all DGAT2s, which is consistent with previous reports indicating that these two highly conserved residues may be located at the active sites of the enzymes and make significant contributions to their enzymatic activities [49]. The phylogenetic analysis of the HpDGAT2s and other DGAT orthologues from eukaryotic algae and plants is illustrated in Additional file 8: Fig. S4, which is consistent with most previous results [2026]. Briefly, all HpDGAT2s clustered with the algal DGAT2s orthologues, which are distinct from other DGAT subfamilies, including DGAT1, DGAT3, and DGAT/WSD. Of the five HpDGAT2s, HpDGAT2A formed a monophyletic subgroup (BS: 100\%) with CrDGAT2A, CzDGAT2A, CzDGAT2B, LiDGAT2A, and LiDGAT2B. HpDGAT2B and HpDGAT2E were highly close (BS: $98 \%$ ) to CrDGAT2B, CzDGAT2E and CrDGAT2C. HpDGAT2C was evolutionarily close (BS: $100 \%$ ) to CzDGAT2C and LiDGAT2C. HpDGAT2D built a monophyletic subgroup (BS: 73\%) with CrDGAT2D and CzDGAT2D.

\section{AST and TAG accumulation and HpDGAT2s gene transcription upon exposure to high light and nitrogen deficient stresses}

High light (HL) and nitrogen deficient (nitrogen-free, ND) stresses can effectively promote the accumulation of AST and TAG in H. lacustris [32-34, 50-53]. However, under such circumstances, the growth of algae was completely restricted [51-53]. Recently, our team completed research investigating the effects of nitrogen deficiency (nitrogen content compared to growth in control BBM medium, e.g., $0,1 / 4 \mathrm{~N}, 1 / 2 \mathrm{~N}$, and $3 / 4 \mathrm{~N}$ ) on algal growth and AST and TAG accumulation. The results indicated that the highest AST productivity was achieved under $1 / 4 \mathrm{~N}$ stress due to a certain level of algal growth. Therefore, in the current manuscript, the $1 / 4 \mathrm{~N}$ condition was selected as the nitrogen deficient stress for further experiments. To understand the relationship between HpDGAT2s transcription and TAG and AST biosynthesis, time-course patterns of algal biomass, expression, total AST (T-AST), and total TAG (T-TAG) contents in photoautotrophic cultures of $H$. lacustris under $\mathrm{HL}, 1 / 4 \mathrm{~N}$, and double $\mathrm{HL}-1 / 4 \mathrm{~N}$ stresses were studied (Fig. 1).

As shown in Fig. 1a, compared to the control, HL, 1/4 $\mathrm{N}$, and double HL-1/4 N stresses inhibited algal growth. The T-AST production and composition are summarized in Fig. 1b-e. From these results, we could draw the conclusions that (1) M-AST is the main form; (2) 


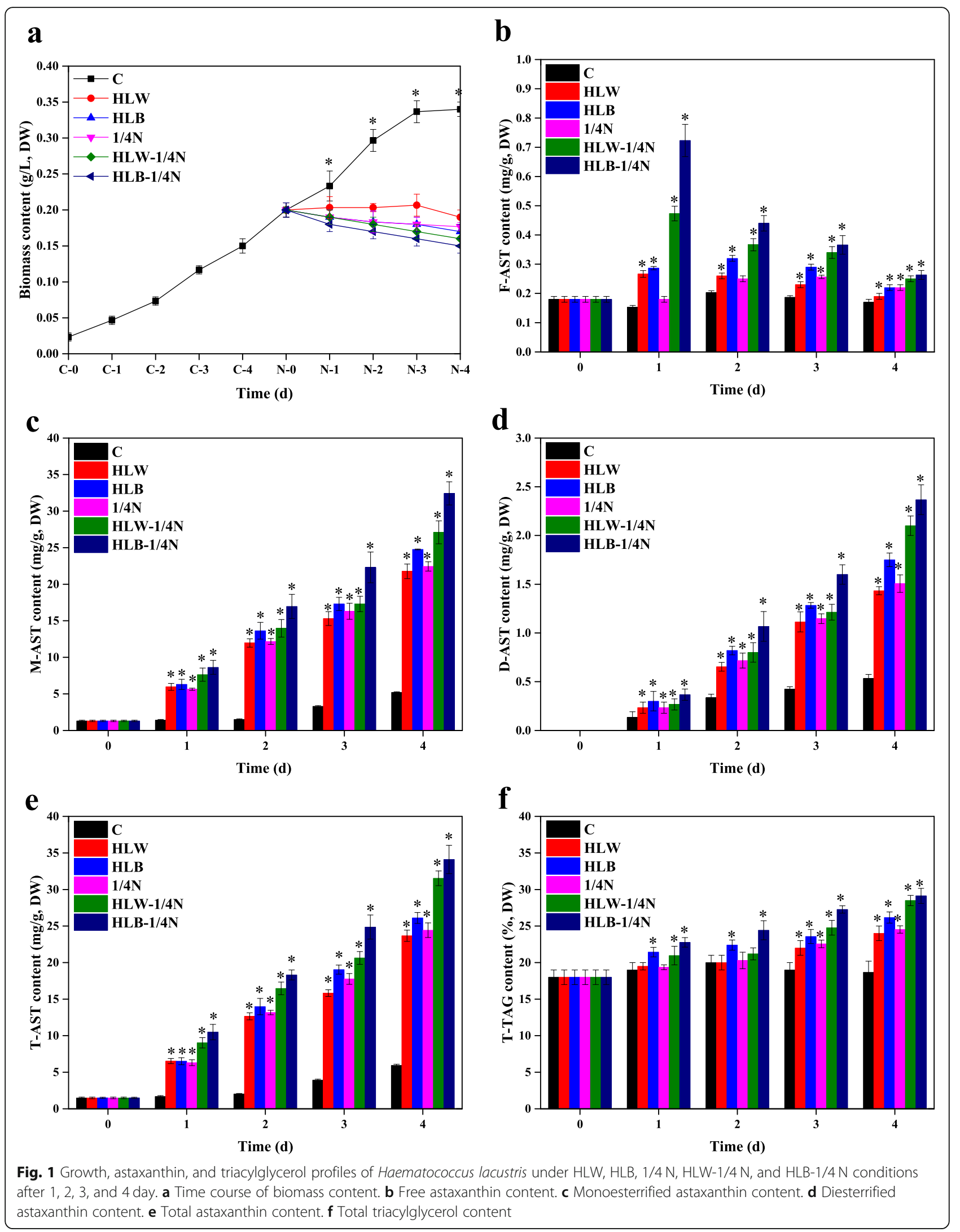


compared to $1 / 4 \mathrm{~N}$ stress, HL is more effective at inducing AST accumulation, especially under high blue light (HLB) conditions; and (3) coupled HL and 1/4 N dual stimulation might be a better choice for improving AST accumulation. Moreover, T-TAG contents slowly increased from day 1 to day 4 and reached maximum values of $29.5,28.7,26.8,25.2$, and $24.8 \%$ under HLB-1/ $4 \mathrm{~N}, \mathrm{HLW}-1 / 4 \mathrm{~N}, \mathrm{HLB}, 1 / 4 \mathrm{~N}$, and HLW conditions, respectively, which were $159.5,155.1,144.9,136.2$, and $134.1 \%$ higher than the values of the control (Fig. 1f). The effects of HL, $1 / 4 \mathrm{~N}$ and double HL-1/4 $\mathrm{N}$ stresses on TAG and AST accumulation were largely consistent with previous studies showing that AST and lipid biosynthesis were enhanced and that the former was coordinated with later biosynthesis under $\mathrm{HL}$ and ND conditions [34, 41]. Previous studies have indicated that DGAT enzymes are probably responsible for both AST esterification and TAG biosynthesis in H. lacustris [33, 34]. As revealed by qRT-PCR results (Fig. 2), the HpDGAT2 gene transcription expression levels exhibited distinct patterns under $\mathrm{HL}, 1 / 4 \mathrm{~N}$ and double HL-1/4 $\mathrm{N}$ stresses. Of the five $H p D G A T 2 s$, the $H p D G A T 2 B$ and $H p D G A T 2 C$ expression levels decreased and remained constant (Fig. 2b and c). The HpDGAT2A and HpDGAT2E expression levels increased and reached their maximum at $4 \mathrm{~d}$ of exposure, and they were $\mathrm{HL}$ and $1 / 4 \mathrm{~N}$ stress-dependent (Fig. 2a and e), respectively, while the HpDGAT2D expression level increased and was stress dependent (Fig. 2d). These results suggested that these HpDGAT2A, $H p D G A T 2 D$, and HpDGAT2E genes were together involved in AST and TAG biosynthesis under stress.

\section{Functional complementation of HpDGAT2s in yeast}

To verify the function of the putative HpDGAT2s enzymes, the ORF-encoding sequences were cloned (Additional file 4: Table S4) into the pYES2.0 plasmid and heterologously expressed in the quadruple mutant yeast

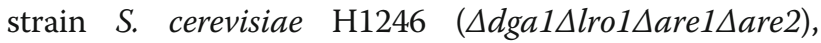
which lacks TAG synthesis activity. Mutant type (H1246) yeast can form TAG when at least one of these four genes is expressed. Furthermore, wild-type (INVSc1) and H1246-EV (H1246 harbouring empty vector pYES2.0) yeast strains were used as positive and negative controls, respectively.

The expression of HpDGAT2A, HpDGAT2B, $H p D G A T 2 D$, and HpDGAT2E restored TAG biosynthesis at different levels in H1246 cells, as indicated by the remarkable TAG spot on a TLC plate (Fig. 3a). In contrast, HpDGAT2B expression in H1246 cells produced inconspicuous TAG levels, indicating a nonfunctional encoded protein considering the low transcription expression levels in H1246 cells (Fig. 3b) and H. lacustris cells (Fig. 2b). Nevertheless, the limited FA composition in Saccharomyces cerevisiae might lead to low TAG content for HpDGAT2B. The ability of HpDGAT2A, HpDGAT2B, HpDGAT2D, and HpDGAT2E to restore TAG biosynthesis in yeast led us to examine FA substrate specificity. As indicated in Fig. $3 \mathrm{~b}$ and $\mathrm{c}$, the HpDGAT2A, HpDGAT2B, HpDGAT2D, and HpDGAT2E genes were heterologously expressed in H1246 and INVSc1 cells. The changes in TAG content and FA composition of TAGs extracted from the transformed H1246 and INVSc1 cells were similar. As shown in Fig. 3d, the TAG contents of expressed $H p D G A T 2 A$ and $H p D G A T 2 B$ in $\mathrm{H} 1246$ cells were 78.3 and $56.5 \%$ lower, respectively, than those of the control (INVSc1 and INVSc1 + EV). The TAG contents of expressed HpDGAT2D and HpDGAT2E were 108.7 and $122.7 \%$ higher, respectively, than the control. To further test FA substrate specificity, FAs from transformed H1246 and INVSc1 cells were analyzed by GC. As shown in Fig. 3d, compared to the control, the MUFAs palmitoleic acid (C16:1) and oleic acid (C18:1) abundances increased in HpDGAT2A-, $H p D G A T 2 D$-, and HpDGAT2E-expressing H1246 cells at the expense of saturated fatty acids (SFAs), including palmitic acid (C16:0) and stearic acid (C18:0). Such a tendency, however, at different levels was observed for almost all transformed lines of H1246 for various DGAT enzymes [20, 23-28].

Considering the limited FA composition in yeast strains (C16:0, C18:0, C16:1, and C18:1), some PUFAs enriched in $H$. lacustris, including linoleic acid (C18:2n6), $\alpha$-linolenic acid (C18:3n3), $\gamma$-linolenic acid (C18:3n6), and parinaric acid $(\mathrm{C} 18: 4 \mathrm{n} 3)$, were tested for substrate specificity for the HpDGAT2A, HpDGAT2B, HpDGAT2D, and HpDGAT2E enzymes by employing a feeding strategy. HpDGAT2A, HpDGAT2D, and HpDGAT2E had similar tendencies to incorporate these PUFAs into TAG at the expense of $\mathrm{C} 16: 1$ and $\mathrm{C} 18: 1$ with the following patterns: C18: 2n6 > C18:3n3 > C18:3n6 > C18:4n3 (Fig. 3e). Considering that $\mathrm{C} 18: 2 \mathrm{n} 6$ and $\mathrm{C} 18: 3 \mathrm{n} 3$ were rich in $H$. lacustris, it is reasonable to speculate that these HpDGAT2s may have potential in C18:2n6- and C18: 3n3-enriched TAG production [32-34]. The HpDGAT2A, HpDGAT2D, and HpDGAT2E enzymes showed a stronger preference for PUFAs than MUFAs due to the higher feeding content of PUFAs than endogenous MUFAs content. This phenomenon was also confirmed by Zienkiewicz et al. (2018), who incorporated some PUFAs into TAG at the expense of 16:1 and 18:1 in LiDGAT1-, LiDGAT2.1-, LiDGAT2.2-, and LiDGAT2.3-expressing yeast [23] and in CzDGAT2C-expressing mutant $\mathrm{H} 1246$ yeast cells [26] by feeding tests. However, FA profiles of the TAG fraction from yeast cells expressing $H p D G A T 2 B$ showed no obvious changes, implying a nonfunctional protein (Fig. 3e). 


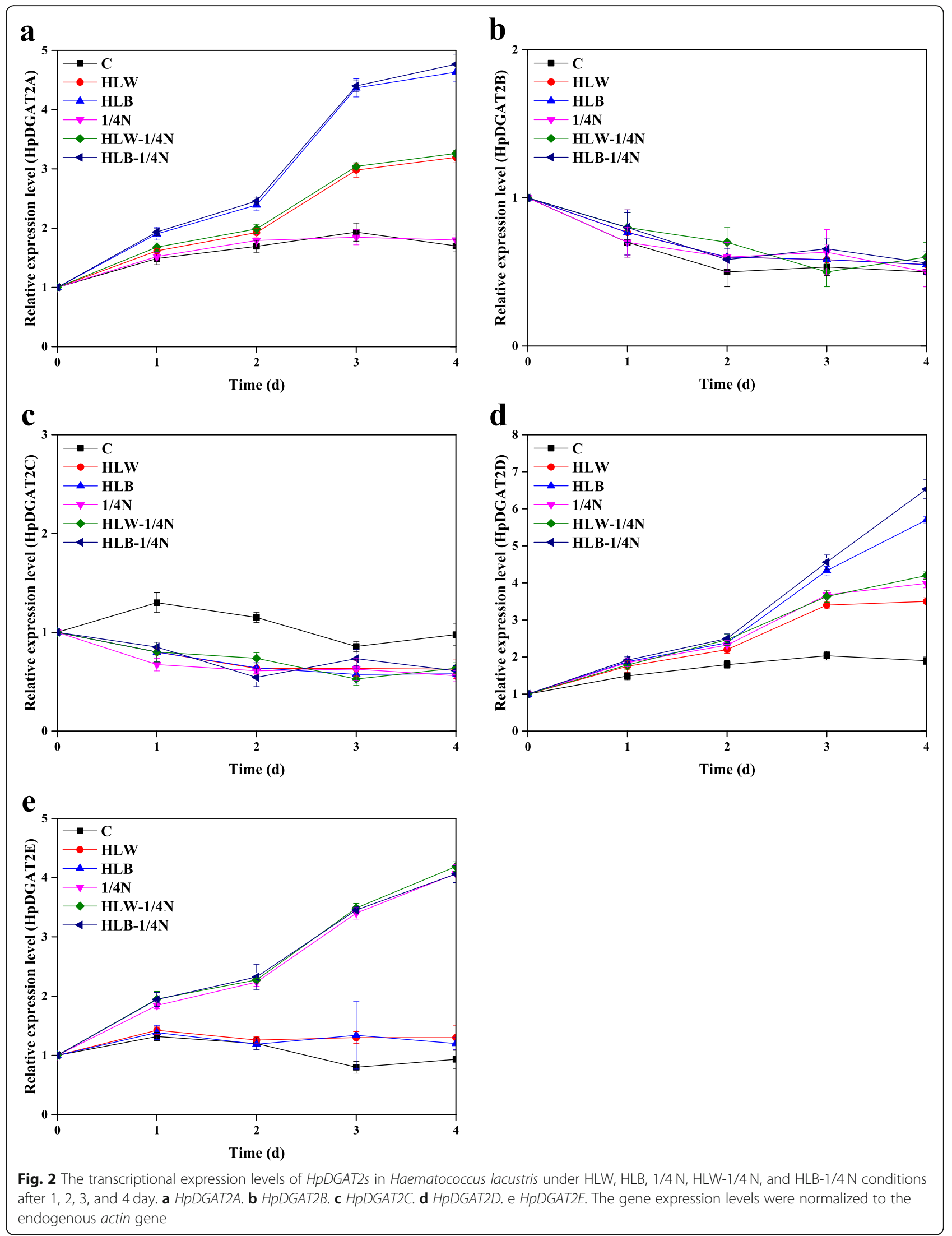




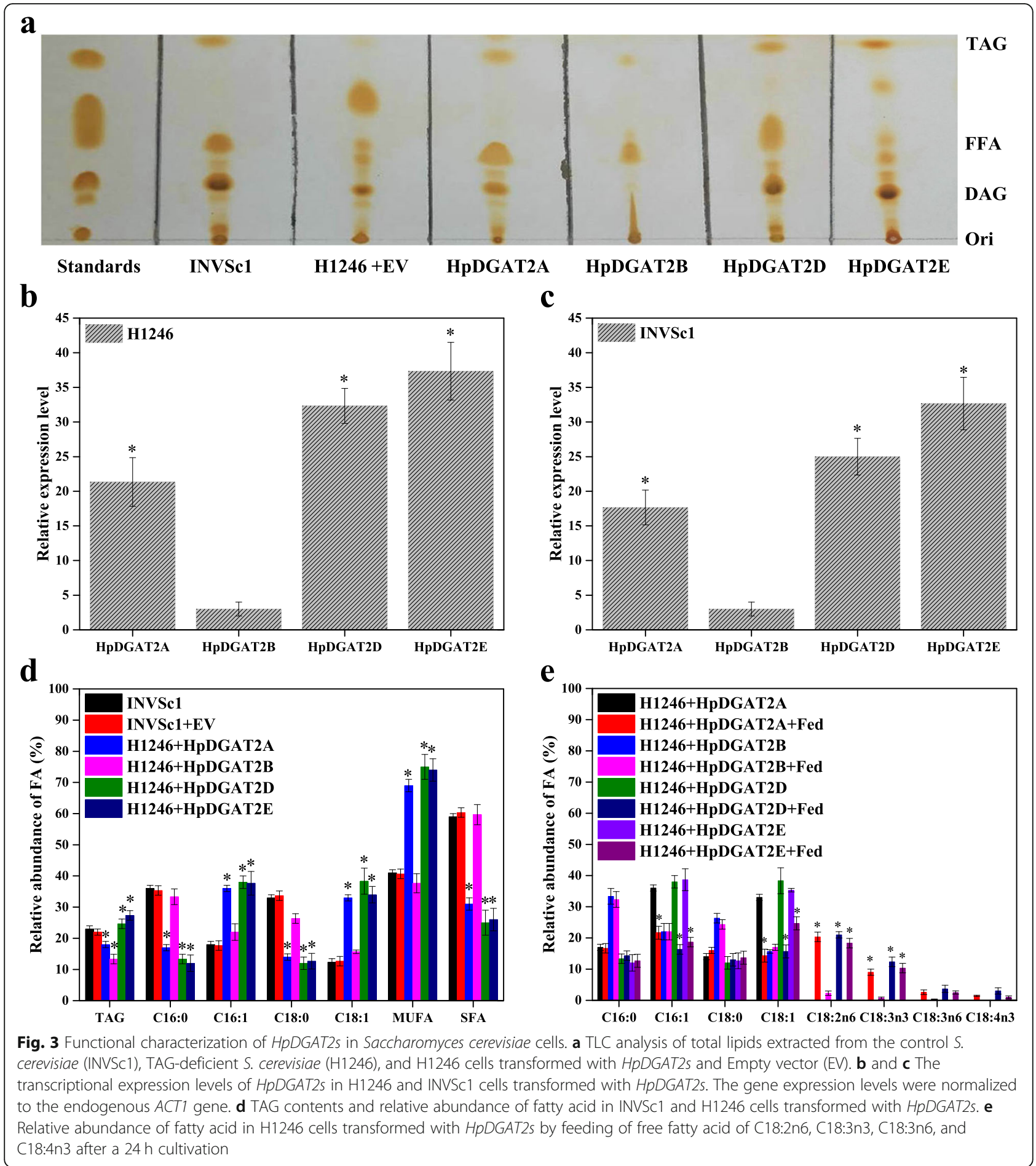

HpDGAT2D heterologous expression promotes TAG biosynthesis and its relative MUFAs and PUFAs abundance in $C$. reinhardtii

To investigate the possible biological role of HpDGAT2s and their engineering potential to modulate TAG biosynthesis in algae, we generated $H p D G A T 2 D$ heterologous expression lines in the evolutionarily close green alga $C$. reinhardtii CC849. HpDGAT2D was selected for further experiments due to the relatively strong TAG biosynthetic activity in yeast cells (Fig. 3) and high transcription expression level in $H$. lacustris under stress conditions (Fig. 2d).

The nuclear transformation expression vector pDB124 (Additional file 9: Fig. S5), characterized in C. reinhardtii 
CC849 and gifted by professor Zhangli Hu from Shenzhen University, was used in this study after modification because it contained overexpression cassettes of the $H p D G A T 2 D-H i s$ fusion and bleomycin resistance Ble genes under the control of the verified endogenous promoter and terminator of the PsaD and RBCS2 genes, respectively (Fig. 4a). The codon preference (HpDGAT2D) was optimized according to the alga $C$. reinhardtii (Additional file 10: Fig. S6) before constructing the expression vector. Transformants (screening over 20 putative transformants) were selected on TAP plates supplemented with bleomycin and confirmed by genomic PCR. The exogenous $H p D G A T 2 D-H i s$ fusion gene was integrated into the alga chromosome due to the clear band using the HpDGAT2D$\mathrm{Cr}$ gene as primers in transformation lines, whereas no signal was detected in WT cells (Fig. 4b and Additional file 11: Fig. S7a). Three heterologous expression lines, HpDGAT2D-4, HpDGAT2D-7, and HpDGAT2D-9, exhibited a maximum increase in transcription levels (by $~ 5.5$-fold higher than the control) under ND conditions in a 4-day batch culture, with no significant difference in cell growth between the transgenic lines and the control (Fig. 4c and d). Furthermore, in vivo heterologous expression of the HpDGAT2D protein was validated by using His-tagged antibodies via western blot analysis. Bands were present in the membrane proteins of three heterologous expression lines (HpDGAT2D-4, $H p D G A T 2 D-7$, and HpDGAT2D-9), but were absent from the soluble proteins, which was consistent with HpDGAT2D being a transmembrane enzyme (Fig. 4e and Additional file 11: Fig. S7b). HpDGAT2D heterologous expression led to considerable increases (by $\sim 1$.4-fold) in TAG content under ND conditions (Fig. 4f). HpDGAT2D heterologous expression also affected the FA profiles in TAGs (Fig. 4f). A significant increase was observed in the relative abundance of MUFAs (C16:1 and C18:1) and PUFAs (C18:2n6 and $\mathrm{C} 18: 3 \mathrm{n} 3$ ), accompanied by a significant decrease in SFAs (C16:0 and C18:0) and some PUFAs (C16:2, C16:3, C18:3n6, and C18:4n3). These results indicated that (1) HpDGAT2D showed a stronger preference for MUFAs and PUFAs than SFAs; (2) of all PUFAs, HpDGAT2D chose C18:2n6 and C18:3n3 as the first option rather than C16:2, C16:3, C18: 3n6, and C18:4n3; and (3) these preferred substrates were enriched in C. reinhardtii. This trend was consistent with results from yeast cells obtained by feeding test (Fig. 3d and e) and previous studies of NoDGAT1A expression in C. reinhardtii UVM4 and CzDGAT1A expression in oleaginous alga N. oceanica by Wei et al. (2017) and Mao et al. (2019), respectively [20, 22].

\section{HpDGAT2D heterologous expression enhances seed oil content and its relative MUFAs and PUFAs abundance in A. thaliana}

To explore HpDGAT2s as a tool to manipulate acylCoA pools and to engineer TAG biosynthesis in higher plants, $H p D G A T 2 D$ was heterologously expressed in Arabidopsis thaliana. Three $A$. thaliana independent expression T2 generation lines (At-HpDGAT2D-3, At$H p D G A T 2 D-6$, and At-HpDGAT2D-8) were selected for further detailed analysis. There were no visible morphological difference (e.g., 1000-seed weight) between the transgenic lines and untransformed control $A$. thaliana (Fig. 5a). The qRT-PCR results showed that the $H p D G A T 2 D$ transcript was expressed in transgenic lines in different tissue organs, including roots, tubers, leaves, siliques, and seeds, to different extents (Fig. 5b). The transformation of wild-type $A$. thaliana with HpDGAT2D resulted in higher (120.0-126.4\%) seed TAG content than the control (Fig. 5c). Again, further GC analysis of FA profiles from TAGs revealed that PUFAs and MUFAs significantly increased, accompanied by a significant decrease in SFAs (Fig. 5c). However, the exact alteration process was much more complicated than those in yeast and C. reinhardtii cells. Specifically, of the SFAs, C16:0 and C22:0 decreased while C18:0 and C20:0 remained stable. Of MUFAs and PUFAs, HpDGAT2D preferred C18:1, C18:2n6, and C18:3n3 rather than C20:1, C20:2 and C22:1 in TAG biosynthesis. These results were largely in agreement with those from yeast cells (Fig. 3d and e) and C. reinhardtii cells (Fig. 4c). Guo et al. (2017) indicated that the CeDGAT1 gene can stimulate FA biosynthesis and enhance seed weight and oil content when expressed in A. thaliana and B. napus [21].

\section{Discussion}

Usually, the accumulation of AST and TAG is simultaneously significantly enhanced under most stress conditions in H. lacustris, e.g., HL and ND conditions [29-35, 50-53]. However, in general, nitrogen deficiency seriously limits algal growth [51-53]. Recently, our results indicated that the highest AST productivity was achieved under $1 / 4 \mathrm{~N}$ stress based on a certain level of algal growth. Therefore, in the current manuscript, the $1 / 4 \mathrm{~N}$ condition was selected as the ND condition in further experiments. Our results revealed that (1) T-AST and TTAG contents significantly increased under HL and $1 / 4$ $\mathrm{N}$ conditions, respectively, which was consistent with some previous studies [34, 41]; (2) M-AST was the main form, which has also been proven by previous studies [36-39]; (3) compared to $1 / 4 \mathrm{~N}$ stress, HL was more effective in inducing AST accumulation, especially under high blue light conditions, which was demonstrated in our previous study [50]; and (4) coupled HL and 1/4 N dual stimulation might be better choices for AST and TAG accumulation in H. lacustris (Fig. 1) [53]. Although the specific mechanisms of stress-induced TAG and AST accumulation in $H$. lacustris are largely unknown, several lines of evidence have implied that the biosynthesis of TAG and AST appears to be linked to the 


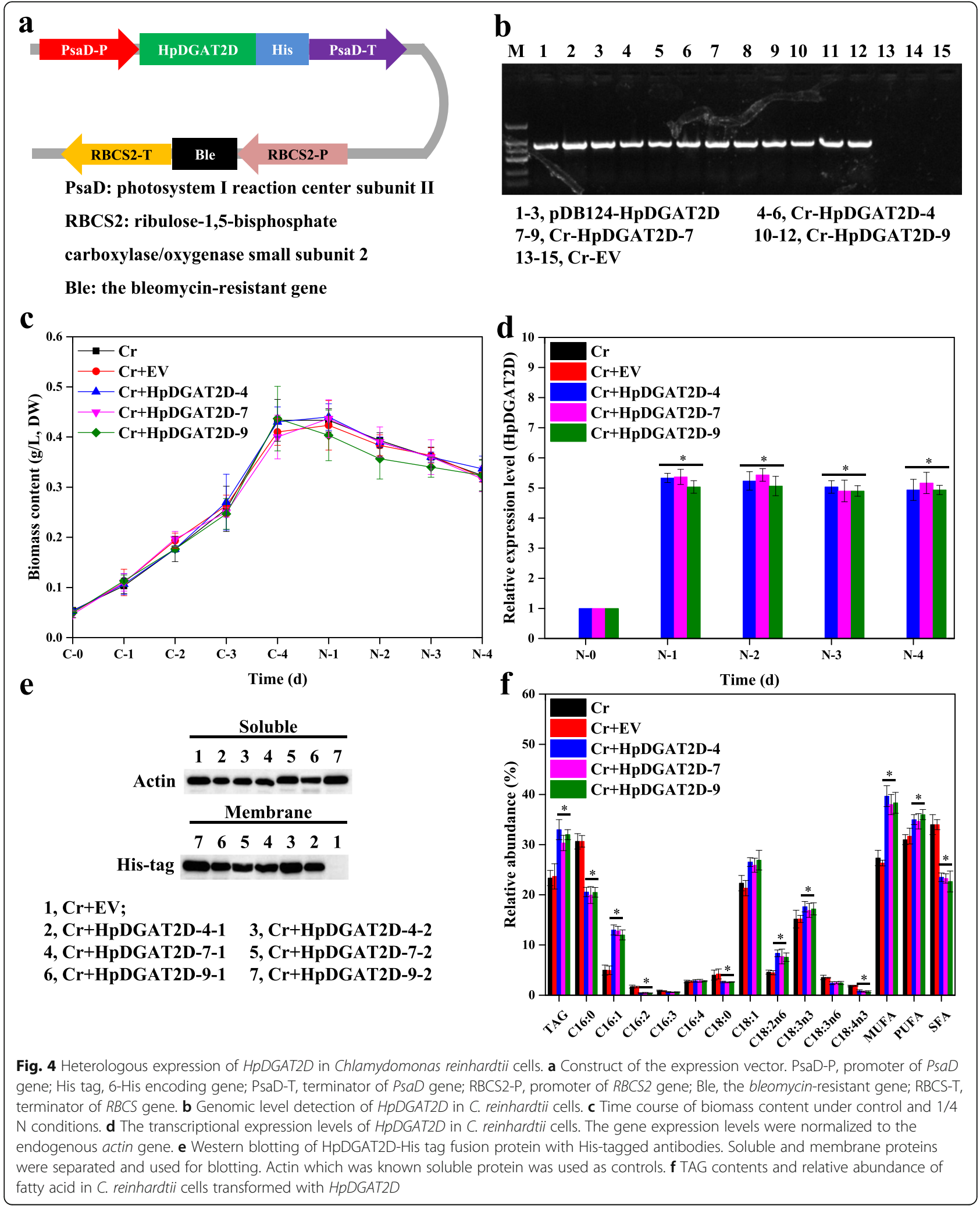



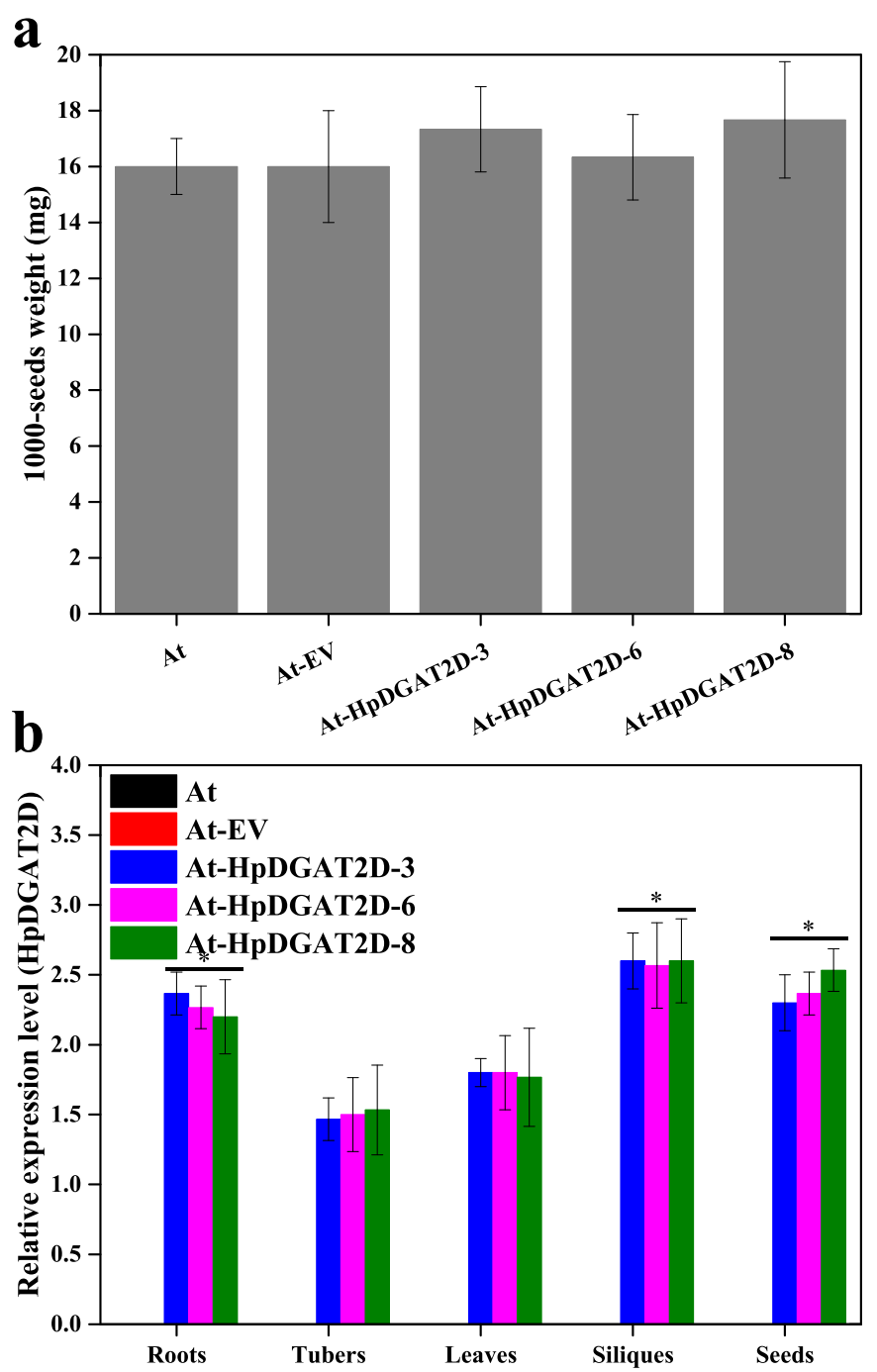

c

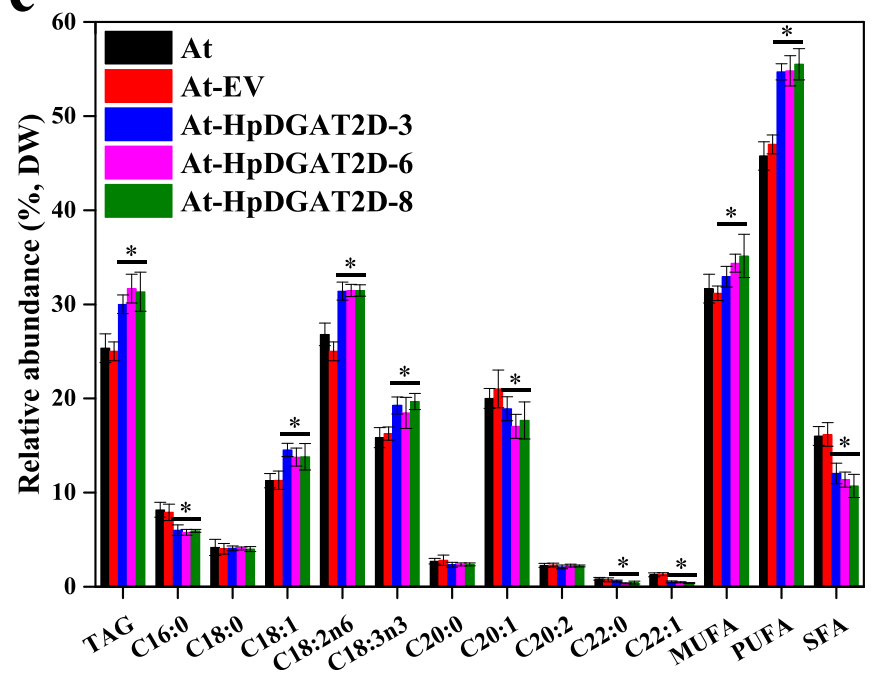

Fig. 5 Heterologous expression of HpDGAT2D in Arabidopsis thaliana. a Average 1000-seed weight (expressed as milligrams of weight/1000 seeds) of transgenic Arabidopsis T2 seeds. $\mathbf{b}$ The transcriptional expression levels of HpDGAT2D in A. thaliana. The gene expression levels were normalized to the endogenous actin gene. c TAG contents and relative abundance of fatty acid in A. thaliana transformed with HpDGAT2D 
regulation of oil biosynthetic enzymes at the transcription level [34, 41]. In fact, AST accumulation is dependent on FA biosynthesis and TAG accumulation in H. lacustris [34, 41]. Recently, Zhang et al. (2019) reported that synthesized AST was esterified mainly with the fatty acid C18:1 and stored in TAG-filled lipid droplets in C. zofingiensis [40]. Unlike in H. lacustris, although AST accumulated in a well-coordinated manner with TAG, AST is ketolated from zeaxanthin and is independent of FA synthesis in C. zofingiensis [40]. This contrasting result may be due to the differences in the genetic traits of these two organisms. The enzymes involved in EAST biosynthesis in the AST-producing algae H. lacustris and C. zofingiensis are unclear.

DGATs catalyze the terminal step in the acyl-CoAdependent TAG production pathway and represent key targets for manipulating TAG production [11]. At present, DGATs from different algal species have been widely studied, which indicates that diverse microalgae are prominent candidates for DGATs and that the function of distinct DGATs is unique or species-specific [19-28]. Obviously, the HpDGAT2 genes were differentially regulated by $\mathrm{HL}, 1 / 4 \mathrm{~N}$, and double $\mathrm{HL}-1 / 4 \mathrm{~N}$ stress conditions with distinct patterns, suggesting that these enzymes are together involved in AST and TAG biosynthesis (Fig. 2). Mao et al. (2019) indicated that CzDGAT1A, CzDGTT1, CzDGTT5 and CzDGTT8 were all considerably up-regulated by ND with distinct expression patterns [20]. Chen et al. (2015) indicated that the transcript level of MiDGAT2A was regulated by ND stress, which led to TAG accumulation [28]. In addition, previous studies have indicated that DGATs are possible candidate enzymes involved in both TAG and EAST accumulation [34], which makes it more interesting to identify DGATs in the AST-producing industrial alga $H$. lacustris [29]. Recently, although four putative type-2 DGAT genes were identified from $H$. pluvialis (lacustris), and only HpDGAT2D had the ability to restore TAG biosynthesis in a TAG-deficient yeast strain [42], the engineering potential of DGAT2s in TAG biosynthesis remains ambiguous.

In this study, we demonstrated that there were five DGAT2s genes in the alga $H$. lacustris, which we renamed HpDGAT2A, HpDGAT2B, HpDGAT2C, HpDGAT2D, and $H p D G A T 2 E$ according to sequence alignment and phylogenetic analysis results (Additional file 3: Table S3 and Additional file 8: Fig. S4), updating a previous report of four putative type-2 DGATs in the H. pluvialis (lacustris) transcriptome database [42]. Generally, only one or two copies of DGAT1s are present in a number of microalgae, whereas multiple copies of DGAT2s are typically present [14]. The number of DGAT2s is species-specific in various algal organisms, e.g., Chlamydomonas reinhardtii (5), Nannochloropsis oceanica (13), Lobosphaera incise (3),
Chlorella zofingiensis (8), Myrmecia incise (2), and Phaeodactylum tricornutum (4) [20, 23, 24, 26-28]. Subcellular localization prediction revealed the different sub-locations of HpDGAT2s (Additional file 2: Table S2), which is consistent with the subcellular localization prediction of DGATs from the green algae C. reinhardtii [24] and $C$. zofingiensis [20]. Two or three TMs were present in all HpDGAT2s (Additional file 2: Table S2 and Additional file 5: Fig. S1), implying they were members of the membrane-bound forms of DGAT1 and DGAT2 [14]. Interestingly, abundant phosphorylation sites were predicted in all HpDGAT2s (Additional file 2: Table S2 and Additional file 6: Fig. S2), indicating that phosphorylation plays important roles in DGAT2s enzyme activity, given that DGAT1 enzyme activity is affected by phosphorylation of mouse DGAT1 [44], BnaDGAT1 [46] and TmaDGAT1 [45]. It remains to be determined whether these phosphorylation sites are important for the functional regulation of HpDGAT2 in vivo. The CDs previously identified in DGAT2 enzymes from higher plants and microalgae [26, 47, 48] were also present in HpDGAT2s but with varying degrees of conservation (Additional file 7: Fig. S3), including YF/YFP block (CD1), which is essential for DGAT2 activity; HPHG/EPHS block (CD4), which is proposed to partially consist of the active site; and $\operatorname{RxGFx}(\mathrm{K} /$

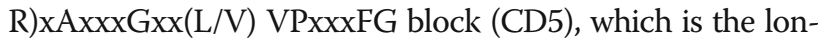
gest conserved sequence in plants and animals. Some putative lipid binding motifs (FLxLxxx and FVLF blocks) in mouse DGAT2 were not conserved among HpDGAT2s and algal DGAT2s $[47,48,54]$. Moreover, there were two completely conserved amino acid residues (proline, $\mathrm{P}$ and phenylalanine, F) among all DGAT2s, which is consistent with previous reports that these two highly conserved residues may be located at the active sites of the enzymes [49].

To characterize the roles of HpDGAT2s, four $H p D G A T 2 s$ genes with full-length coding sequences (Additional file 2: Table S2) were heterologously expressed in the TAG-deficient yeast strain H1246 [55]. The results indicated that all of the HpDGAT2s genes are functional with the large differences in enzymatic activity (Fig. 3a). Further functional characterization in yeast showed that HpDGAT2D and HpDGAT2E can increase the TAG content more than HpDGAT2A and HpDGAT2B, resulting in a significant increase in the TAG content of yeast by $108.7-122.7 \%$ (Fig. 3d). This higher activity provides an alternative candidate for DGAT2 to modulate TAG accumulation in algae. However, a previous study detected that only HpDGAT2D had the ability to restore TAG biosynthesis in a TAGdeficient yeast strain [42]. In contrast, in our study, $H p D G A T 2 B$ expression in $\mathrm{H} 1246$ cells produced inconspicuous TAG, possibly due to the limited FAs in Saccharomyces cerevisiae. This holds true, at least for CzDGTT1 expressed in yeast, as the TAG content 
increased when feeding on two other free FAs [20]. It is also possible that HpDGAT2B may not be a real DGAT but another type of transferase, which cannot be differentiated based only on the protein sequence [20]. This phenomenon is usually present in green algae, e.g., CrDGTT1 through CrDGTT3 are functional, while CrDGTT4 is not [24]. NoDGAT1A and CzDGTT1, rather than NoDGAT1B, are functional [20, 22].

DAGs and fatty acyl-CoAs are essential substrates for TAG biosynthesis under the catalysis of DGAT enzymes $[20,22,24]$. The fatty acyl-CoA substrate specificity was determined by FA profile analysis. HpDGAT2s showed a strong preference for MUFAs (C16:1 and C18:1) in yeast cells. Such a tendency, however, at different levels was observed for almost all transformed lines of H1246 for various DGAT enzymes [20, 23, 24, 26-28]. Considering the limited FAs in yeast cells, some PUFAs (e.g., C18: 2n6, C18:3n3, C18:3n6, and C18:4n3) that are present in $H$. lacustris but not in yeast cells were selected to test the acyl-CoA substrate specificity by using a feeding strategy. Interestingly, all HpDGAT2s except for HpDGAT2B showed a wide range of preference for PUFAs with distinct patterns in yeast cells, especially for C18:2n6 and C18:3n3, which are also rich in H. lacustris, indicating that these HpDGAT2s may have potential for the engineering of PUFAs-enriched TAG production. This phenomenon was also confirmed by Zienkiewicz et al. (2018), who incorporated some PUFAs into TAG at the expense of C16:1 and C18:1 in LiDGAT1-, LiDGAT2.1-, LiDGAT2.2-, and LiDGAT2.3-expressing yeast [23] and CzDGAT2C-expressing yeast mutant H1246 cells [26] by feeding tests. Consistent with the low transcription of $H p D G A T 2 B$ in algal and yeast cells, the feeding test demonstrated the low preference of PUFAs, again indicating a nonfunctional encoded protein. Although the acyl-CoA substrate preference was characterized, the DAG (prokaryotic and eukaryotic) substrate specificity needs to be elucidated in the future.

To evaluate the possible biological function and engineering potential of HpDGAT2s to modulate TAG biosynthesis in algae and plants, in the present study, we generated heterologous expression lines in the evolutionarily close green alga C. reinhardtii CC849 and the model plant $A$. thaliana. It is not surprising that HpDGAT2D heterologous expression enhanced TAG contents in both $C$. reinhardtii CC849 (by $\sim 1.4$-fold) and $A$. thaliana (by $\sim 1.2$-fold). Guo et al. (2017) indicated that the CeDGAT1 gene can stimulate FA biosynthesis and enhance seed weight and oil content when expressed in A. thaliana and B. napus [21]. Compared to the control, under $1 / 4 \mathrm{~N}$ stress conditions, it was also worth noting that the TAG content was significantly increased in a 4-day batch culture for HpDGAT2D heterologous expression lines under the same stress conditions (Fig. 4b), possibly due to the high transcription level (Fig. 4d). Wei et al. (2017) detected that, under nitrogen-replete conditions, NoDGAT1A expression in C. reinhardtii UVM4 had no effect on TAG accumulation, while TAG enhancement was observed under nitrogen-depleted conditions [22]. However, Mao et al. (2019) declared that CzDGAT1A expression in the oleaginous alga $N$. oceanica resulted in a considerable increase ( 2.8-fold) in TAG levels [20]. Consistent with the strong preference for MUFAs and PUFAs rather than SFAs in yeast cells, HpDGAT2D also showed a similar trend in C. reinhardtii. Specifically, HpDGAT2D first opted for C16:1, C18:1, C18:2n6 and C18:3n3 rather than C16:2, C16:3, C16:4, C18:3n6, and C18:4n3. Interestingly, these preferred substrates were enriched in $C$. reinhardtii, indicating their potential for the engineering of $C$. reinhardtii for MUFAs- and PUFAs-enriched TAG production. This trend was also consistent with results from yeast cells in feeding tests (Fig. $3 \mathrm{~d}$ and e) and consistent with previous studies of NoDGAT1A expression in $C$. reinhardtii UVM4 and CzDGAT1A expression in oleaginous alga $N$. oceanica by Wei et al. (2017) and Mao et al. (2019), respectively [20, 22]. In higher plants, the expression of DGATs generally enhances oil deposition in developing seeds [56]. For example, stronger expression of DGAT1 was detected in developing seeds than in other tissues in soybeans [57]. However, DGAT1 transcripts were also present in other plant tissues, although they were strongest in developing embryos and flower petals [58]. In the current study, the HpDGAT2D transcript was heterologously expressed in transgenic lines at different tissue organs, including roots, tubers, leaves, siliques, and seeds, to different extents (Fig. 5b). However, the exact process of FA change was much more complicated than those in yeast and C. reinhardtii cells (Fig. 5c). HpDGAT2D showed a strong preference for C18:1, C18:2n6, and C18:3n3 rather than C20:1, C20: 2 and C22:1 in TAG biosynthesis, which was largely in agreement with the preference in yeast cells (Fig. $3 \mathrm{~d}$ and e) and C. reinhardtii cells (Fig. 4c). Previous studies have indicated that seed-specific overexpression of EgDGAT2 in A. thaliana enhanced the content of PUFAs C18:2n6 and C18:3n3 in seed TAG when compared to that from wild-type Arabidopsis. In turn, the proportion of C18:0 and C20:0 SFAs in seed TAG from EgDGAT2 transgenic lines decreased accordingly [59]. In Thraustochytrium aureum, DGAT2 expression under a strong seed-specific promoter in wild-type $A$. thaliana increased C18:2n6 content [60]. In addition, transgenic plants showed no other phenotypic differences. Therefore, HpDGAT2D should have great potential for increasing the specific oil production in other oil crops.

Although it has been previously suggested that DGATs may be involved in the esterification of AST in $H$. 
lacustris [34], there is no direct biochemical evidence to support this hypothesis. Recently, all $10 \mathrm{CzDGATs}$ were expressed in a reconstructed AST-producing yeast strain [61] to examine whether these enzymes were responsible for EAST biosynthesis. However, no EAST was detected, indicating the null function of CzDGATs in AST esterification [20]. Considering the differences in genetic traits and AST biosynthetic pathways of both AST-producing algal strains, C. zofingiensis and $H$. lacustris, we will study the possible roles of HpDGAT2s in AST esterification in the future.

\section{Conclusions}

Here, we performed an in-depth characterization of HpDGAT2s by integrating expression patterns, AST/ TAG accumulation, functional complementation, and heterologous expression in yeast, plants, and algae. Five putative DGAT2s genes (HpDGAT2A, HpDGAT2B, $H p D G A T 2 C, H p D G A T 2 D$, and HpDGAT2E) were identified in $H$. lacustris by BLAST and CD analysis. These $D G A T 2 s$ genes showed markedly increased transcription levels under stress conditions, which led to significant TAG and EAST accumulation. Functional complementation demonstrated that HpDGAT2A, HpDGAT2B, HpDGAT2D, and HpDGAT2E had the ability to restore TAG synthesis in a TAG-deficient yeast strain (H1246) with a large difference in enzymatic activity. FA profile assays revealed that HpDGAT2A, HpDGAT2D, and HpDGAT2E, but not HpDGAT2B, preferred MUFAs for TAG synthesis in yeast cells and showed PUFAs preference by feeding strategy. The heterologous expression of $H p D G A T 2 D$ in wild-type $A$. thaliana and $C$. reinhardtii significantly increased the TAG content and showed a strong preference for MUFAs and PUFAs, indicating the engineering potential to increase specific TAG production in plants and algae.

\section{Methods}

\section{Algal strain and growth conditions}

The unicellular algal Haematococcus lacustris/pluvialis (FACHB-712) strain was obtained from the Freshwater Algae Culture Collection at the Institute of Hydrobiology and maintained at the Institute of Molecular Agriculture and Bioenergy (IMAB), Shanxi Agricultural University. $H$. lacustris was cultivated in $100-\mathrm{mL}$ of BBM medium in 250-mL Erlenmeyer flasks. These Erlenmeyer flasks were placed under culture conditions of $25 \mu \mathrm{mol} / \mathrm{m}^{2} / \mathrm{s}$ light intensity with a diurnal cycle of $12 \mathrm{~h}$ light $/ 12 \mathrm{~h}$ dark at $23 \pm 1{ }^{\circ} \mathrm{C}$. The culture solution was shaken for a fixed time twice a day. For the HL treatment, after the cultures were dark-adapted for $48 \mathrm{~h}$, the later exponentially growing cultures (biomass content of approximately $200 \mathrm{mg} / \mathrm{L}$ ) were further transferred into fresh medium under continuous white light (390-770 $\mathrm{nm})$ or blue light $(420-500 \mathrm{~nm})$ with a light intensity of $500 \mu \mathrm{mol} / \mathrm{m}^{2} / \mathrm{s}$ without a light/dark cycle. For the $1 / 4 \mathrm{~N}$ nitrogen deficient treatment, the pre-cultured and darkinduced cells were collected and washed with nitrogenfree $B B M$ medium and then further transferred into fresh $B B M$ medium with $1 / 4$ nitrogen content (the same as that in the BBM medium) under control culture conditions without a light/dark cycle. For $\mathrm{HL}$ and $1 / 4 \mathrm{~N}$ double stress $(\mathrm{HL}-1 / 4 \mathrm{~N})$ treatment, pre-cultured and dark-induced cells were transferred into fresh medium with $1 / 4$ nitrogen content under the same continuous white light or blue light. The cultures under control conditions were used as the control samples. These precultured and dark-induced cells, after centrifugation and washing with sterilized water, were sampled as the starting point ( $\mathrm{N}-0$ day). The cultures were sampled $\mathrm{N}-1, \mathrm{~N}-$ $2, \mathrm{~N}-3$, and N-4 days after treatment. The cells were harvested by centrifugation $\left(13,100 \mathrm{~g}\right.$ at $4{ }^{\circ} \mathrm{C}$ for $\left.5 \mathrm{~min}\right)$ and washed with PBS prior to storage in liquid nitrogen. For cell dry biomass determination, $20 \mathrm{~mL}$ of cell culture was collected and washed three times, and then the EP tubes containing cells were dried in a DW3 freeze-drier (Heto Dry Winner, Denmark).

\section{Cloning and bioinformatics analysis of HpDGAT2s}

The genes encoding putative HpDGAT2s were predicted and cloned as follows: (1) the local BLAST program was used to predict DGAT2s genes based on the $H$. lacustris transcriptome database with annotated CzDGAT2s and CrDGAT2s (Additional file 1: Table S1), (2) the rapid amplification of cDNA ends (RACEs) method was used to obtain the full-length mRNA sequences and then determine their transcription start sites, stop sites, and encoding sequences, and (3) the open reading frame (ORF) for each HpDGAT2s gene was obtained by PCR again to construct distinct expression plasmids. All the primers used in this study are listed in Additional file 4: Table S4. The molecular weight (Mw), isoelectronic point (pI), subcellular localization, signal peptides (SP), chloroplast transfer peptides (CTP), transmembrane regions (TM), and phosphorylation site (Phos) of HpDGAT2s were predicted by Compute pI/MW, TargetP, ChloroP, SignalP, TMHMM, and NetPhos tools, respectively, in ExPASy [62]. HpDGAT2s and other DGATs from plants and algae were aligned using ClustalX [63]. Maximum likelihood trees of HpDGAT2s and other DGAT proteins were constructed using PhyML with the bootstrap (BS) values inferred from 400 replicates $[64,65]$. Graphical representation and editing of the phylogenetic tree were performed with MEGA5 [66] and TreeDyn (v198.3) [67].

\section{RNA isolation and quantitative real-time PCR}

Total RNA was extracted according to the EasySpin RNA Extraction Kit (Aidlab Biotech, Beijing, China) and was quantified by a NanoDrop 2000c (Thermo Scientific, 
USA). The first-strand cDNAs were synthesized according to the instruction of PrimeScript ${ }^{\circ}$ RT Enzyme Mix I (TaKaRa DRR047A, China) Kit. The qRT-PCR was performed as described by our previous study using a 7500 Fast Real-Time PCR System (Applied Biosystems, Waltham, MA, USA) with SYBR Green PCR Master Mix (Invitrogen) [50]. The mRNA expression level was normalized using the actin gene as the internal control. All analyses were based on the CT values of the PCR products. The comparative CT method was used to investigate the transcriptional expression levels of $H p D G A T 2 s$ genes [68].

\section{Functional complementation of HpDGAT2s in the TAG- deficient yeast $\mathrm{H} 1246$}

The ORFs of HpDGAT2A, HpDGAT2B, HpDGAT2D, and $H p D G A T 2 E$ were PCR-amplified using cDNA as a template and cloned into the yeast expression vector pYES2.0 (Invitrogen). After confirmation by restriction enzyme digestion and sequencing, the recombinant pYES2.0-HpDGAT2s plasmids were transformed into the S. cerevisiae TAG-producing strain INVSc1 or TAGdeficient quadruple mutant strain H1246 with the S.c. EasyComp Transformation Kit (Invitrogen) [20]. The expression of $H p D G A T 2$ genes in the yeast strain was verified at the transcript level by qRT-PCR. For the feeding experiments, yeast cultures were induced as described above but in the presence of $1 \%(\mathrm{w} / \mathrm{v})$ Tergitol NP-40 (Sigma Aldrich, St. Louis, MO, USA) in the medium. At the beginning of induction, the appropriate FAs (C18: 2n6, C18:3n3, C18:3n6, and C18:4n3) were added to the culture to a final concentration of $100 \mu \mathrm{M}$. Samples at an OD600 of 2.5 were harvested for lipid extraction, separation by TLC and analysis by GC.

\section{Heterologous expression of HpDGAT2D in C. reinhardtii}

The nuclear transformation expression vector pDB124 (Additional file 9: Fig. S5), characterized in C. reinhardtii CC849 and gifted by professor Zhangli Hu from Shenzhen University [69], was used in this study after modification. The codon preference (HpDGAT2D) was optimized according to the alga $C$. reinhardtii (Additional file 10: Fig. S6) before constructing the expression vector. The codon preference optimized coding sequence of $H p D G A T 2 D$ was amplified and cloned into the PmlI and BmtI sites of pDB-124, followed by sequencing for verification. The resulting plasmid was linearized by $X b a \mathrm{I}$ and transformed into the $C$. reinhardtii cc849 strain via the glass beads method [70]. Transformants were selected on Tris-acetate-phosphate (TAP) plates with $10 \mu \mathrm{g} / \mathrm{mL}$ bleomycin (Sigma-Aldrich). For ND stress, the later exponentially growing $C$. reinhardtii cc849 cells (biomass content of approximately $420 \mathrm{mg} /$ L) were used following the methods described in the above section. The integration of HpDGAT2D into the
Chlamydomonas genome was verified by genomic PCR, and its transcription and protein expression levels were determined by qRT-PCR and western blotting using histagged antibodies, respectively. Considering that HpDGAT2D was a transmembrane protein, soluble and membrane proteins from HpDGAT2D-His fusionheterologous expressing $C$. reinhardtii cells were used for immunodetection as previously described [19].

\section{Heterologous expression of HpDGAT2D in A. thaliana}

The coding sequence of $H p D G A T 2 D$ was amplified and cloned into EcoRI/XbaI sites of pCAMBIA1303 to yield pCAMBIA1303-HpDGAT2D. After verified by restriction enzyme digestion and sequencing, the pCAMBIA1303-HpDGAT2D vector was firstly transferred into Agrobacterium tumefaciens strain GV3101 [71], and finally transferred into $A$. thaliana plants by vacuum infiltration [72]. T1 generation seeds were selected on hygromycin $(50 \mathrm{mg} / \mathrm{L})$ and $\mathrm{T} 2$ transgenic A. thaliana lines were used for further analyses. The stable integration of pCAMBIA1301-HpDGAT2D into the genome and the transcription expression were determined by genomic PCR and qRT-PCR, respectively.

\section{Total astaxanthin analysis}

The HPLC method was applied to quantify the contents of different AST forms using the standard curve of AST (purchased from Sigma-Aldrich) at known concentrations [50, 73].

\section{Lipid extraction and fatty acid analysis}

Total lipids extraction, TAGs separation, and FAs analysis were performed according to previously described procedures [21, 74-76]. Briefly, $50 \mathrm{mg}$ of yeast cells, 10 $\mathrm{mg}$ of freeze-dried algae cells or $10 \mathrm{mg}$ of dried seeds were used to extract total lipids according to previously reported methods [75]. Then, TAGs were separated by thin-layer chromatography (TLC) methods as descripted in previous study [21]. Finally, TAGs were transesterified with $5 \% \mathrm{H}_{2} \mathrm{SO}_{4}$ in methanol at $85^{\circ} \mathrm{C}$ for $1 \mathrm{~h}$ and the fatty acid methyl esters (FAMEs) were analyzed by an Agilent GC equipped with a flame ionization detector (FID) and a capillary column (HP-88100 $\mathrm{m} \times 0.25$ $\mathrm{mm} \times 0.2 \mathrm{~mm}$ ) with an appropriate add amount of C17:0 FAME (Sigma) as an internal standard [75].

\section{Statistical analysis}

All experiments were repeated three times to ensure reproducibility. The data were obtained as the mean value \pm SD. Statistical analyses were performed using the SPSS statistical package (SPSS Inc., Chicago, IL, USA). Significant differences between treatments were statistically analyzed by paired-samples t-test. Statistical significance was achieved when $P<0.01$. 


\section{Supplementary Information}

The online version contains supplementary material available at https://doi. org/10.1186/s12870-020-02794-6.

Additional file 1 Table S1. GenBank accession numbers (from National Center for Biotechnology Information) for DGAT and ACTIN proteins sequence used in this study. Note: Cz, Chromochloris zofingiensis; No, Nannochloropsis oceanica; Cr, Chlamydomonas reinhardtii; Pt, Phaeodactylum tricornutum; Li, Lobosphaera incise; At, Arabidopsis thaliana; Gm, Glycine max; Zm, Zea mays; Sc, Saccharomyces cerevisiae.

Additional file $\mathbf{2}$ Table $\mathbf{S 2}$. Gene sequences information and biochemical features of HpDGAT2s in Haematococcus lacustris. Note: anformation regarding HPDGAT2C is predicted based on the partial coding sequence obtained from the transcriptome database.

Additional file $\mathbf{3}$ Table S3. Amino acid sequence identity (\%; blue) and similarity (\%; red) between HpDGAT2s and CrDGAT2s.

Additional file $\mathbf{4}$ Table S4. Primers used in this study.

Additional file 5 Figure S1. Predicated trans-membrane domains for HpDGAT2A, HpDGAT2B, HpDGAT2C, HpDGAT2D, and HpDGAT2E by TMHMM v. 2.0 Server (http://www.cbs.dtu.dk/services/TMHMM-2.0/

Additional file 6 Figure S2. Predicated phosphorylation site for HpDGAT2A, HpDGAT2B, HpDGAT2C, HpDGAT2D, and HpDGAT2E by NetPhos 3.1 Server (http://www.cbs.dtu.dk/services/NetPhos/)

Additional file $\mathbf{7}$ Figure S3. Proteins sequences alignment of putative HpDGAT2s and other annotated DGAT2s from plants and microalgae. Proteins sequences with accession number used in this study were listed in in Additional file 1: Table S1. Blue color indicates the key conserved domains and black asterisk indicates the key amino acid residues.

Additional file 8 Figure S4. Phylogenetic analysis of HpDGAT2s and other annotated DGATs from higher plants and microalgae. Protein sequences used in this study were listed in Additional file 1: Table S1.

Additional file 9 Figure S5. A schematic map of the pDB124HpDGAT2D vector. It contained an expression cassette of the HPDGAT2D gene under the control of the endogenous and characterized PsaD promoter and PsaD terminator, an expression cassette of the Ble gene controlled by the endogenous and characterized RBCS2 promoter and RBCS2 terminator, and an expression cassette of the Amp resistance gene, which conferred resistance to ampicillin.

Additional file 10 Figure S6. Original sequences of HpDGAT2D in Haematococcus lacustris and new sequences of HpDGAT2D-Cr after codon optimization for Chlamydomonas reinhardtii. Red color stands for the modified nucleotide sequence.

Additional file 11 Figure S7. Genomic level of HpDGAT2D in C. reinhardtii cells (a) and western blotting of HpDGAT2D-6-His tag fusion protein with His-tag antibody (b). Note: Soluble and membrane proteins were separated and used for blotting. Actin which was known soluble protein was used as controls. M, DNA marker or protein marker (Nonwestern blotting type).

\section{Abbreviations}

AST: Astaxanthin; E-AST: Esterified astaxanthin; F-AST: Free AST; MAST: Momoester AST; D-AST: Diester AST; TAG: Triacylglycerol; DGAT: Diacylglycerol acyltransferase; ORF: Open reading frame; RACEs: Rapid amplification of complementary DNA ends; UTR: Untranslated region; HL: High light; ND: Nitrogen deficient; MUFAs: Monounsaturated fatty acylCoAs; PUFAs: Polyunsaturated fatty acyl-CoAs

\section{Acknowledgements}

We acknowledge all the members of the research team for their assistance in the field and laboratory work. We thank Professor Hongyan Zhu from University of Kentucky for helping to polish the language and Professor Zhangli Hu from Shenzhen University for gifting pDB124 plasmid.

\section{Authors' contributions}

$\mathrm{HC}$ and $\mathrm{CZ}$ carried out the experiments, analyzed the data and drafted the manuscript. WX and $\mathrm{HZ}$ performed the over-expression of HpDGAT2s in yeast, algae and plants. WH and XZ participated in gene cloning and sequence analysis of HpDGAT2s. CJ and JX participated in transcription expression of HpDGAT2s in $\mathrm{H}$. lacustris under stress condition. CZ participated in growth and TAG and AST accumulation for $H$. lacustris under stress condition. $\mathrm{RL}$ conceived the study, participated in its design and revised the manuscript. All authors read and approved the final manuscript.

\section{Funding}

This study was supported by the National Natural Science Foundation of China (31902394), Key Research and Development Planning Project of Shanxi Province (201803D31063), Applying Basic Research Planning Project of Shanxi Province (201801D221250), Key Research and Development Planning Project of Jinzhong City (Y192012), Science and Technology Innovation Planning Project of Shanxi Agricultural University (2018YJ16), Shanxi Province Key Projects of Coal-based Science and Technology (FT-2014-01), Shanxi Scholarship Council of China (2015-064), and the Key Project of the Key Research and Development Program of Shanxi Province, China (201603D312005). The funding bodies were not involved in the design of the study, collection, analysis, and interpretation of data, and in writing the manuscript.

\section{Availability of data and materials}

The datasets supporting the results of this article are included within the article and its additional files. The five DGATs proteins (HpDGAT2A: MT875161; HpDGAT2B: MT875162; HpDGAT2C: MT875163; HpDGAT2D: MT875164; HpDGAT2E: MT875165) from Haematococcus lacustris have been deposited in NCBI database. All the other raw data of protein sequences used in the current study were downloaded from the NCBI database (https://www.ncbi.nlm.nih.gov/protein/?term=dgat) and the GenBank accession numbers were listed in Additional file 1: Table S1.

\section{Ethics approval and consent to participate}

Not applicable.

\section{Consent for publication}

Not applicable.

\section{Competing interests}

The authors declare that they have no competing interests.

Received: 1 February 2020 Accepted: 9 December 2020

Published online: 06 January 2021

\section{References}

1. Giorno F, Mazzei R, Giorno L. Purification of triacylglycerols for biodiesel production from Nannochloropsis microalgae by membrane technology. Bioresour Technol. 2013;140:172-8.

2. Bilal M, Rasheed T, Ahmed I, labal HMN. High-value compounds from microalgae with industrial exploitability - a review. Front Biosci. 2017:9:319-42.

3. Hempel F, Maier UG. Microalgae as solar-powered protein factories. Adv Eep Med Biol. 2016;896:241-62

4. Ma H, Xiong H, Zhu X, Ji C, Xue J, Li R, et al. Polysaccharide from Spirulina platensis ameliorates diphenoxylate-induced constipation symptoms in mice. Int J Biol Macromol. 2019:133:1090-101.

5. Ma H, Chen S, Xiong H, Wang M, Hang W, Zhu X, et al. Astaxanthin from Haematococcus pluvialis ameliorates the chemotherapeutic drug (doxorubicin) induced liver injury through the Keap1/Nrf2/HO-1 pathway in mice. Food Funct. 2020;11:4659-71.

6. Chen Y, Xu C, Vaidyanathan S. Microalgae: a robust "green bio-bridge" between energy and environment. Crit Rev Biotechnol. 2018;38:351-68.

7. Lenka SK, Carbonaro N, Park R, Miller SM, Thorpe I, Li Y. Current advances in molecular, biochemical, and computational modeling analysis of microalgal triacylglycerol biosynthesis. Biotechnol Adv. 2016;34:1046-63.

8. Goncalves EC, Wilkie AC, Kirst M, Rathinasabapathi B. Metabolic regulation of triacylglycerol accumulation in the green algae: identification of potential targets for engineering to improve oil yield. Plant Biotechnol J. 2016;14: 1649-60.

9. Xue J, Niu YF, Huang T, Yang WD, Liu JS, Li HY. Genetic improvement of the microalga Phaeodactylum tricornutum for boosting neutral lipid accumulation. Metab Eng. 2015;27:1-9. 
10. Bates PD, Browse J. The significance of different diacylgycerol synthesis pathways on plant oil composition and bioengineering. Front Plant Sci. 2012;3:147.

11. Bhatt-Wessel B, Jordan TW, Miller JH, Peng L. Role of DGAT enzymes in triacylglycerol metabolism. Arch Biochem Biophys. 2018;655:1-11.

12. Chen JE, Smith AG. A look at diacylglycerol acyltransferases (DGATs) in algae. J Biotechnol. 2012;162:28-39.

13. Liu Q, Siloto RM, Lehner R, Stone SJ, Weselake RJ. Acyl-CoA:diacylglycerol acyltransferase: molecular biology, biochemistry and biotechnology. Prog Lipid Res. 2012;51:350-77.

14. Xu Y, Caldo KMP, Pal-Nath D, Ozga J, Lemieux MJ, Weselake RJ, et al. Properties and biotechnological applications of acyl-CoA:diacylglycerol acyltransferase and phospholipid:diacylglycerol acyltransferase from terrestrial plants and microalgae. Lipids. 2018;53:663-88.

15. Bagnato C, Prados MB, Franchini GR, Scaglia N, Miranda SE, Beligni MV. Analysis of triglyceride synthesis unveils a green algal soluble diacylglycerol acyltransferase and provides clues to potential enzymatic components of the chloroplast pathway. BMC Genomics. 2017;18:223.

16. Cui Y, Zhao J, Wang Y, Qin S, Lu Y. Characterization and engineering of a dual-function diacylglycerol acyltransferase in the oleaginous marine diatom Phaeodactylum tricornutum. Biotechnol Biofuels. 2018;11:32.

17. Li F, Wu X, Lam P, Bird D, Zheng H, Samuels L, et al. Identification of the wax ester synthase/acyl-coenzyme a: diacylglycerol acyltransferase WSD1 required for stem wax ester biosynthesis in Arabidopsis. Plant Physiol. 2008 148:97-107.

18. Hernández ML, Whitehead L, He Z, Gazda V, Gilday A, Kozhevnikova E, et al. A cytosolic acyltransferase contributes to triacylglycerol synthesis in sucroserescued Arabidopsis seed oil catabolism mutants. Plant Physiol. 2012;160: 215-25.

19. La Russa M, Bogen C, Uhmeyer A, Doebbe A, Filippone E, Kruse O, et al. Functional analysis of three type-2 DGAT homologue genes for triacylglycerol production in the green microalga Chlamydomonas reinhardtii. J Biotechnol. 2012;162:13-20.

20. Mao X, Wu T, Kou Y, Shi Y, Zhang Y, Liu J. Characterization of type I and type II diacylglycerol acyltransferases from the emerging model alga Chlorella zofingiensis reveals their functional complementarity and engineering potential. Biotechnol Biofuels. 2019;12:28.

21. Guo X, Fan C, Chen Y, Wang J, Yin W, Wang RR, et al. Identification and characterization of an efficient acyl-CoA:diacylglycerol acyltransferase 1 (DGAT1) gene from the microalga Chlorella ellipsoidea. BMC Plant Biol. 2017; 17:48

22. Wei H, Shi Y, Ma X, Pan Y, Hu H, Li Y, et al. A type-l diacylglycerol acyltransferase modulates triacylglycerol biosynthesis and fatty acid composition in the oleaginous microalga, Nannochloropsis oceanica. Biotechnol Biofuels. 2017;10:174.

23. Zienkiewicz K, Benning U, Siegler H, Feussner I. The type 2 acyl-CoA: diacylglycerol acyltransferase family of the oleaginous microalga Lobosphaera incisa. BMC Plant Biol. 2018;18:298.

24. Liu J, Han D, Yoon K, Hu Q, Li Y. Characterization of type 2 diacylglycerol acyltransferases in Chlamydomonas reinhardtii reveals their distinct substrate specificities and functions in triacylglycerol biosynthesis. Plant J. 2016;86:3-19.

25. Guihéneuf F, Leu S, Zarka A, Khozin-Goldberg I, Khalilov I, Boussiba S. Cloning and molecular characterization of a novel acyl-CoA:diacylglycerol acyltransferase 1-like gene (PtDGAT1) from the diatom Phaeodactylum tricornutum. FEBS J. 2011;278:3651-66

26. Xu Y, Falarz L, Chen G. Characterization of type-2 diacylglycerol acyltransferases in the green microalga Chromochloris zofingiensis. J Agr Food Chem. 2019;67:291-8.

27. Gong Y, Zhang J, Guo X, Wan X, Liang Z, Hu CJ, et al. Identification and characterization of PtDGAT2B, an acyltransferase of the DGAT2 acylcoenzyme a:diacylglycerol acyltransferase family in the diatom Phaeodactylum tricornutum. FEBS Lett. 2013;587:481-7.

28. Chen CX, Sun Z, Cao HS, Fang FL, Ouyang LL, Zhou ZG. Identification and characterization of three genes encoding acyl-CoA:diacylglycerol acyltransferase (DGAT) from the microalga Myrmecia incisa Reisigl. Algal Res. 2015;12:280-8

29. Khoo KS, Lee SY, Ooi CW, Fu X, Miao X, Ling TC, et al. Recent advances in biorefinery of astaxanthin from Haematococcus pluvialis. Bioresour Technol. 2019;288:121606

30. Solovchenko AE. Recent breakthroughs in the biology of astaxanthin accumulation by microalgal cell. Photosynth Res. 2015;125:437-49.
31. Shah MM, Liang Y, Cheng JJ, Daroch M. Astaxanthin-producing green microalga Haematococcus pluvialis: from single cell to high value commercial products. Front Plant Sci. 2016;7:531.

32. Lei $A$, Chen $H$, Shen $G, H u$ Z, Chen L, Wang J. Expression of fatty acid synthesis genes and fatty acid accumulation in Haematococcus pluvialis under different stressors. Biotechnol Biofuels. 2012;5:18.

33. Ma R, Thomas-Hall SR, Chua ET, Alsenani F, Eltanahy E, Netzel ME, et al. Gene expression profiling of astaxanthin and fatty acid pathways in Haematococcus pluvialis in response to different LED lighting conditions. Bioresour Technol. 2018;250:591-602.

34. Chen G, Wang B, Han D, Sommerfeld M, Lu Y, Chen F, et al. Molecular mechanisms of the coordination between astaxanthin and fatty acid biosynthesis in Haematococcus pluvialis (Chlorophyceae). Plant J. 2015;81:95-107.

35. Kwan TA, Kwan SE, Peccia J, Zimmerman JB. Selectively biorefining astaxanthin and triacylglycerol co-products from microalgae with supercritical carbon dioxide extraction. Bioresour Technol. 2018;269:81-8.

36. Miao F, Lu D, Li Y, Zeng M. Characterization of astaxanthin esters in Haematococcus pluvialis by liquid chromatography-atmospheric pressure chemical ionization mass spectrometry. Anal Biochem. 2006;352:176-81.

37. Holtin K, Kuehnle M, Rehbein J, Schuler P, Nicholson G, Albert K. Determination of astaxanthin and astaxanthin esters in the microalgae Haematococcus pluvialis by LC-(APCI) MS and characterization of predominant carotenoid isomers by NMR spectroscopy. Anal Bioanal Chem. 2009;395:1613-22.

38. Fábryová T, Tůmová L, Dcd S, Pereira DM, Andrade PB, Valentão P, et al. Isolation of astaxanthin monoesters from the microalgae Haematococcus pluvialis by high performance countercurrent chromatography (HPCCC) combined with high performance liquid chromatography (HPLC). Algal Res. 2020:49:1247.

39. Pick U, Zarka A, Boussiba S, Davidi L. A hypothesis about the origin of carotenoid lipid droplets in the green algae Dunaliella and Haematococcus. Planta. 2019;249:31-47

40. Zhang $Y$, Ye Y, Ding W, Mao X, Li Y. Astaxanthin is ketolated from zeaxanthin independent of fatty acid synthesis in Chromochloris zofingiensis. Plant Physiol. 2020;183:883-97.

41. Zhekisheva M, Zarka A, Khozin-Goldberg I, Cohen Z, Boussiba S. Inhibition of astaxanthin synthesis under high irradiance does not abolish triacylglycerol accumulation in the green alga Haematococcus pluvialis (Chlorophyceae). J Phycol. 2005:41:819-26.

42. Nguyen T, Xu Y, Abdel-Hameed M, Sorensen JL, Singer SD, Chen G. Characterization of a type-2 diacylglycerol acyltransferase from Haematococcus pluvialis reveals possible allostery of the recombinant enzyme. Lipids. 2019;55:425-33.

43. Gao Z, Li Y, Wu G, Li G, Sun H, Deng S, et al. Transcriptome analysis in Haematococcus pluvialis: astaxanthin induction by salicylic acid (SA) and jasmonic acid (JA). PLoS One. 2015;10:e0140609.

44. Yu J, Li Y, Zou F, Xu S, Liu P. Phosphorylation and function of DGAT1 in skeletal muscle cells. Biophys Rep. 2015:1:41-50.

45. Xu J, Francis T, Mietkiewska E, Giblin EM, Barton DL, Zhang Y, et al. Cloning and characterization of an acyl-CoA-dependent diacylglycerol acyltransferase 1 (DGAT1) gene from Tropaeolum majus, and a study of the functional motifs of the DGAT protein using site-directed mutagenesis to modify enzyme activity and oil content. Plant Biotechnol J. 2008;6:799-818.

46. Caldo KMP, Shen W, Xu Y, Hanley-Bowdoin L, Chen G, Weselake RJ, et al. Diacylglycerol acyltransferase 1 is activated by phosphatidate and inhibited by SnRK1-catalyzed phosphorylation. Plant J. 2018;96:287-99.

47. Liu Q, Siloto RM, Snyder CL, Weselake RJ. Functional and topological analysis of yeast acyl-CoA:diacylglycerol acyltransferase 2, an endoplasmic reticulum enzyme essential for triacylglycerol biosynthesis. J Biol Chem. 2011;286:13115-26.

48. Stone SJ, Levin MC, Farese RV Jr. Membrane topology and identification of key functional amino acid residues of murine acyl-CoA:diacylglycerol acyltransferase-2. J Biol Chem. 2006;281:40273-82.

49. Cao H. Structure-function analysis of diacylglycerol acyltransferase sequences from 70 organisms. BMC Res Notes. 2011:4:249.

50. Cui H, Yu X, Wang Y, Cui Y, Li X, Liu Z, et al. Evolutionary origins, molecular cloning and expression of carotenoid hydroxylases in eukaryotic photosynthetic algae. BMC Genomics. 2013;14:457.

51. Ding $W, Z$ Zhao $Y, X u$ JW. Melatonin: a multifunctional molecule that triggers defense responses against high light and nitrogen starvation stress in Haematococcus pluvialis. J Agr Food Chem. 2018;66:7701-11.

52. Recht L, Töpfer N, Batushansky A, Sikron N, Gibon Y, Fait A, et al. Metabolite profiling and integrative modeling reveal metabolic constraints for carbon 
partitioning under nitrogen starvation in the green algae Haematococcus pluvialis. J Biol Chem. 2014;289:30387-403.

53. Zhang WW, Zhou XF, Zhang YL, Cheng PF, Ma R, Cheng WL, et al. Enhancing astaxanthin accumulation in Haematococcus pluvialis by coupled light intensity and nitrogen starvation in column photobioreactors. J Microbiol Biotechnol. 2018;28:2019-28.

54. Zheng L, Shockey J, Bian F, Chen G, Shan L, Li X, et al. Variant amino acid residues alter the enzyme activity of peanut type 2 diacylglycerol acyltransferases. Front Plant Sci. 2017;8:1751.

55. Sandager L, Gustavsson MH, Ståhl U, Dahlqvist A, Wiberg E, Banas A, et al. Storage lipid synthesis is non-essential in yeast. J Biol Chem. 2002;277:6478-82.

56. He X, Turner C, Chen GQ, Lin J, McKeon TA. Cloning and characterization of a cDNA encoding diacylglycerol acyttransferase from castor bean. Lipids. 2004;39:311-8.

57. Li R, Yu K, Hatanaka T, Hildebrand DF. Vernonia DGATs increase accumulation of epoxy fatty acids in oil. Plant Biotechnol J. 2010;8:184-95.

58. Hobbs DH, Lu C, Hills M. Cloning of a cDNA encoding diacylglycerol acyltransferase from Arabidopsis thaliana and its functional expression. FEBS Lett. 1999:452:145-9.

59. Jin Y, Yuan Y, Gao L, Sun R, Chen L, Li D, et al. Characterization and functional analysis of a type 2 diacylglycerol acyltransferase (DGAT2) gene from oil palm (Elaeis guineensis Jacq.) mesocarp in Saccharomyces cerevisiae and transgenic Arabidopsis thaliana. Front. Plant Sci. 2017;8:1791.

60. Zhang C, Iskandarov U, Klotz ET, Stevens RL, Cahoon RE, Nazarenus TJ, et al. A thraustochytrid diacylglycerol acyltransferase 2 with broad substrate specificity strongly increases oleic acid content in engineered Arabidopsis thaliana seeds. J Exp Bot. 2013;64:3189-200.

61. Zhou P, Ye L, Xie W, Lv X, Yu H. Highly efficient biosynthesis of astaxanthin in Saccharomyces cerevisiae by integration and tuning of algal crtZ and bkt. Appl Microbiol Biot. 2015;99:8419-28.

62. Gasteiger E, Gattiker A, Hoogland C, Ivanyi I, Appel RD, Bairoch A. ExPASy: the proteomics server for in-depth protein knowledge and analysis. Nucleic Acids Res. 2003;31:3784-8.

63. Larkin MA, Blackshields G, Brown NP, Chenna R, McGettigan PA, McWilliam H, et al. Clustal W and Clustal X version 2.0. Bioinformatics. 2007;23:2947-8.

64. Le SQ, Lartillot N, Gascuel O. Phylogenetic mixture models for proteins. Philos Trans R Soc B Sci. 2008:363:3965-76.

65. Guindon S, Dufayard JF, Lefort V, Anisimova M, Hordijk W, Gascuel O. New algorithms and methods to estimate maximum-likelihood phylogenies: assessing the performance of PhyML 3.0. Syst Biol. 2010;59:307-21.

66. Tamura K, Peterson D, Peterson N, Stecher G, Nei M, Kumar S. MEGA5: molecular evolutionary genetics analysis using maximum likelihood, evolutionary distance, and maximum parsimony methods. Mol Biol Evol. 2011;28:2731-9.

67. Chevenet F, Brun C, Bañuls AL, Jacq B, Christen R. TreeDyn: towards dynamic graphics and annotations for analyses of trees. BMC Bioinform. 2006;7:439.

68. Livak KJ, Schmittgen TD. Analysis of relative gene expression data using real-time quantitative PCR and the $2\left(^{- \text {Delta Delta } C(T)}\right.$ ) Method. Methods (San Diego, Calif). 2001;25:402-8.

69. Wang YT JXQ, Hu CX, Sun T, Zeng ZY, Cai XQ, et al. Optogenetic regulation of artificial microRNA improves $\mathrm{H}_{2}$ production in green alga Chlamydomonas reinhardtii. Biotechnol Biofuels. 2017;10:257.

70. Kindle KL. High-frequency nuclear transformation of Chlamydomonas reinhardtii. P Natl Acad Sci USA. 1990;87:1228-32.

71. Holsters M, de Waele D, Depicker A, Messens E, van Montagu M, Schell J. Transfection and transformation of Agrobacterium tumefaciens. Mol Gen Genet. 1978;163:181-7.

72. Clough SJ, Bent AF. Floral dip: a simplified method for Agrobacteriummediated transformation of Arabidopsis thaliana. Plant J. 1998;16:735-43.

73. Cui H, Ma H, Cui Y, Zhu X, Qin S, Li R. Cloning, identification and functional characterization of two cytochrome P450 carotenoids hydroxylases from the diatom Phaeodactylum tricornutum. J Biosci Bioeng. 2019;128:755-65.

74. Cui H, Ma H, Chen S, Yu J, Xu W, Zhu X, et al. Mitigating excessive ammonia nitrogen in chicken farm flushing wastewater by mixing strategy for nutrient removal and lipid accumulation in the green alga Chlorella sorokiniana. Bioresour Technol. 2020;303:122940.

75. Liu B, Sun Y, Xue J, Mao X, Jia X, Li R. Stearoyl-ACP ${ }^{\Delta 9}$ desaturase 6 and 8 (GhA-SAD6 and GhD-SAD8) are responsible for biosynthesis of palmitoleic acid specifically in developing endosperm of upland cotton seeds. Front Plant Sci. 2019;10:703.

76. Bligh EG, Dyer WJ. A rapid method of total lipid extraction and purification. Can J Biochem Physiol. 1959:37:911-7.

\section{Publisher's Note}

Springer Nature remains neutral with regard to jurisdictional claims in published maps and institutional affiliations.

Ready to submit your research? Choose BMC and benefit from:

- fast, convenient online submission

- thorough peer review by experienced researchers in your field

- rapid publication on acceptance

- support for research data, including large and complex data types

- gold Open Access which fosters wider collaboration and increased citations

- maximum visibility for your research: over $100 \mathrm{M}$ website views per year

At BMC, research is always in progress.

Learn more biomedcentral.com/submissions 